\title{
Diagnostic Value of Narrow Band Imaging in Visualization of Pathological Lesions in Larynx and Hypopharynx
}

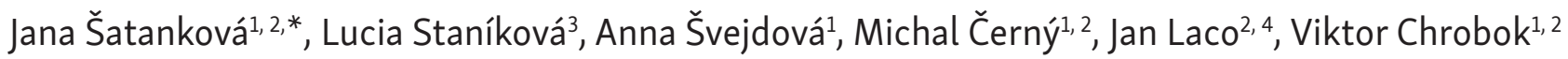

\begin{abstract}
Introduction: Narrow Band Imaging (NBI) is an endoscopic optical imaging enhancement technology that improves the contrast of mucosal surface texture and enhances visualization of mucosal and submucosal vasculature. Due to its properties, it can visualize suspected malignant or precancerous lesions earlier than conventional white light endoscopy. The aim of this study was to analyze the benefit of NBI in visualization of precancerous and malignant lesions in preoperative and intraoperative diagnostics and correlation with histopathologic results.

Methods: A total of 589 patients with suspicious laryngeal or hypopharyngeal lesion were investigated using conventional white light endoscopy (WLE) and NBI endoscopy with high-definition TV (HDTV NBI) from 10/2013 to 12/2019. Patients were divided into two groups based on pre-operative NBI examination (group A, 345 patients) and intraoperative NBI examination (group B, 244 patients). All suspicious lesions were graded to 5 types of Ni classification and correlated with histopathologic results. The SPSS version 8.0.4 statistical software package was used for statistical analysis. In diagnosing premalignant and malignant lesions sensitivity, specificity, positive predictive value, and negative predictive value were calculated.

Results: The agreement between NBI endoscopy and histopathological analysis in group A was statistically significant $(K=0.76, p<0.001)$, with a sensitivity of $86.2 \%$ (95\% IS: $65.4-95.2)$ and specificity of $90.9 \%$ (95\% IS: 70.6-94.1). Moreover, in group B was proven almost perfect agreement between NBI and histopathological analysis $\left(K_{1}=0.8461, p<0.001\right)$, with a sensitivity of $84.0 \%$ (95\% IS: 60.2-92.4) and specificity of $96.0 \%$ (95\% IS: 87.0-99.2).

Conclusions: Based on our results, NBI using the Ni classification has great potential in improving diagnosis of precancerous and malignant lesions and correlates strongly with histopathologic results. It serves as a useful adjunct to white light endoscopy in the diagnosis of laryngeal and hypopharyngeal lesions, especially using HDTV NBI.
\end{abstract}

\section{KEYWORDS}

narrow band imaging; white-light endoscopy; Ni classification; precancerous lesion; squamous cell carcinoma; larynx; hypopharynx

\author{
AUTHOR AFFILIATIONS \\ ${ }^{1}$ Department of Otorhinolaryngology and Head and Neck Surgery, University Hospital Hradec Králové, Czech Republic \\ ${ }^{2}$ Charles University, Faculty of Medicine in Hradec Králové, Czech Republic \\ ${ }^{3}$ Department of Otorhinolaryngology and Head and Neck Surgery, University Hospital Ostrava, Czech Republic \\ ${ }^{4}$ The Fingerland Department of Pathology, University Hospital Hradec Králové, Czech Republic \\ * Corresponding author: Department of Otorhinolaryngology and Head and Neck Surgery, University Hospital Hradec Králové, \\ Czech Republic; e-mail: jana.satankova@fnhk.cz
}

Received: 24 November 2020

Accepted: 12 January 2021

Published online: 14 April 2021

Acta Medica (Hradec Králové) 2021; 64(1): 22-28

https://doi.org/10.14712/18059694.2021.4

(c) 2021 The Authors. This is an open-access article distributed under the terms of the Creative Commons Attribution License (http://creativecommons.org/licenses/by/4.0), which permits unrestricted use, distribution, and reproduction in any medium, provided the original author and source are credited. 


\section{INTRODUCTION}

Squamous cell carcinoma (SCC) is one of the most common types of head and neck cancer. Early detection, histopathological diagnosis and treatment significantly improve prognosis and reduce patient morbidity and mortality (1). The most important limiting factor of the early diagnosis is the fact, that precancerous and early malignant changes in the larynx and hypopharynx do not have specific clinical and macroscopical features different from symptoms and mucosal changes trigerred by common inflammatory disorders (2). Standard examination methods include rigid or flexible laryngoscopy with white light that lacks sensitivity to precancerous (high-grade dysplasia include carcinoma in situ) or early superficial lesions (T1 or T2 carcinoma). Radiologic examinations such as CT and MRI play a complementary role, as they are the only diagnostic tools that are capable of correctly evaluating deep structures, regional and distant lymph node metastases. Growth and progression of SCC relies on neoangiogenesis, a process where new blood vessels are formed from the surrounding pre-existing blood vessels (1). These new vessels lack the histological architecture and structural anatomy of pre-existing vessels that can be detected by conventional white light endoscopy in later stages of cancer. Earlier detection of these changes is very important for better prognosis of the patients (3). Development of new optical endoscopic methods allowed to detect discrete mucosal changes with pathological vascularization and started rapid progress in the diagnostic process of head and neck tumors. One of endoscopic methods that can visualize these changes and improve the diagnosis is NBI, especially HDTV NBI (2).

NBI as a special endoscopic optical imaging enhancement technology improves the contrast of mucosal surface texture and enhances visualization of mucosal and submucosal vasculature by using specially filtered light (blue and green). The blue light (wavelength $415 \mathrm{~nm}$ ) corresponds to hemoglobin absorption spectrum and visualizes IPCL (intraepithelial papillary capillary loops). The green light (wavelength $540 \mathrm{~nm}$ ) penetrates deeper and highlights submucosal vessels. In the definitive image, the mucosal microvascularization appears brown coloured and submucosal vessels are blue coloured (4). Finally, there is a substantial increase of colour contrast between blood vessels and surrounding tissue and a few millimeter changes can be identified. The optimal image is achieved by using HDTV NBI - NBI with high resolution (more than one million pixels) or with ultra high resolution $4 \mathrm{~K}$ (more than 8 million pixels) (2).

Due to its properties, NBI can visualize suspicious malignant or precancerous lesions earlier than conventional WLE. NBI shows great potential by improving detection of tumours in upper aerodigestive tract (UADT) and currently is the most progressive technological tool in diagnosis of head and neck cancer (5).

$\mathrm{Ni}$ et al. proposed a classification system for description of changes in morfology and architecture of IPCL to distinguish between benign and malignant laryngeal lesions (6). This system describes five patterns of morphological changes in IPCL architecture (Table 1). Type I-III are usually associated with benign changes (Figure 1), type IV represents preneoplastic changes and type V neoplastic changes (Figure 2). Type $\mathrm{V}$ is further subdivided into $\mathrm{Va}, \mathrm{Vb}$ a $\mathrm{Vc}$ (Table 1) (5). A new classification system as the result of consensus of European laryngological society was published by Arens et al. in 2016 (7). This system is a simplified version of the Ni classification using a descriptive evaluation of microvascular changes. This classification distinguishes only two types of vascular changes longitudinal and perpendicular (7).

The aim of this study was 1) to evaluate the prehistological diagnostic value of NBI focusing on laryngeal and hypopharyngeal suspicious lesions and 2) to compare NBI with conventional WLE in this setting.

Tab. 1 Classification of intraepithelial papillary capillary loops (Ni et al. 2011).

\begin{tabular}{|l|l|}
\hline Classification & Description \\
\hline Type I & $\begin{array}{l}\text { Thin, oblique and arborescent vessels are inter- } \\
\text { connected } \\
\text { IPCLs are almost invisible }\end{array}$ \\
\hline Type II & $\begin{array}{l}\text { Diameter of oblique and arborescent vessels is } \\
\text { enlarged } \\
\text { IPCLs are almost invisible }\end{array}$ \\
\hline Type III & IPCLs are obscured by white mucosa \\
\hline Type IV & IPCLs can be recognized as small dots \\
\hline Type Va & $\begin{array}{l}\text { IPCLs appear as solid or hollow, with a brownish, } \\
\text { speckled pattern and various shapes }\end{array}$ \\
\hline Type Vb & $\begin{array}{l}\text { IPCLs appear as irregular, tortuous, line - like } \\
\text { shapes }\end{array}$ \\
\hline Type Vc & $\begin{array}{l}\text { IPCLs appear as brownish speckles or tortuous, } \\
\text { line - like shapes with irregular distribution, } \\
\text { scattered on the tumour surface }\end{array}$ \\
\hline
\end{tabular}

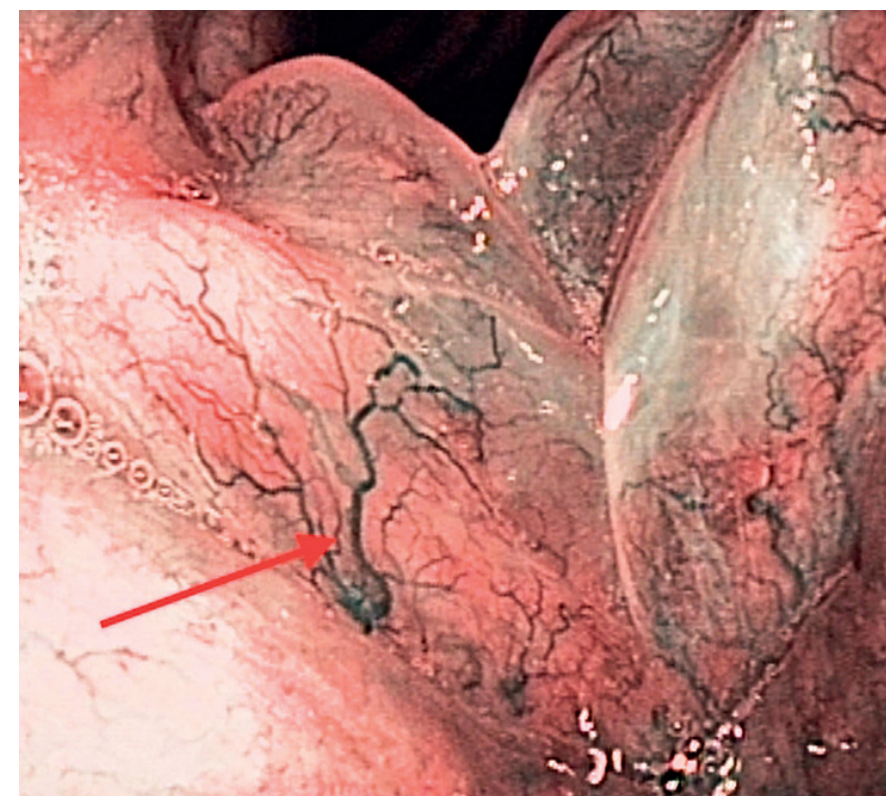

Fig. 1 Reinke oedema of both vocal cords - benign type of vascularization in NBI (the red arrow is showing oblique and arborescent vessels IPCL type II according to Ni classification). 

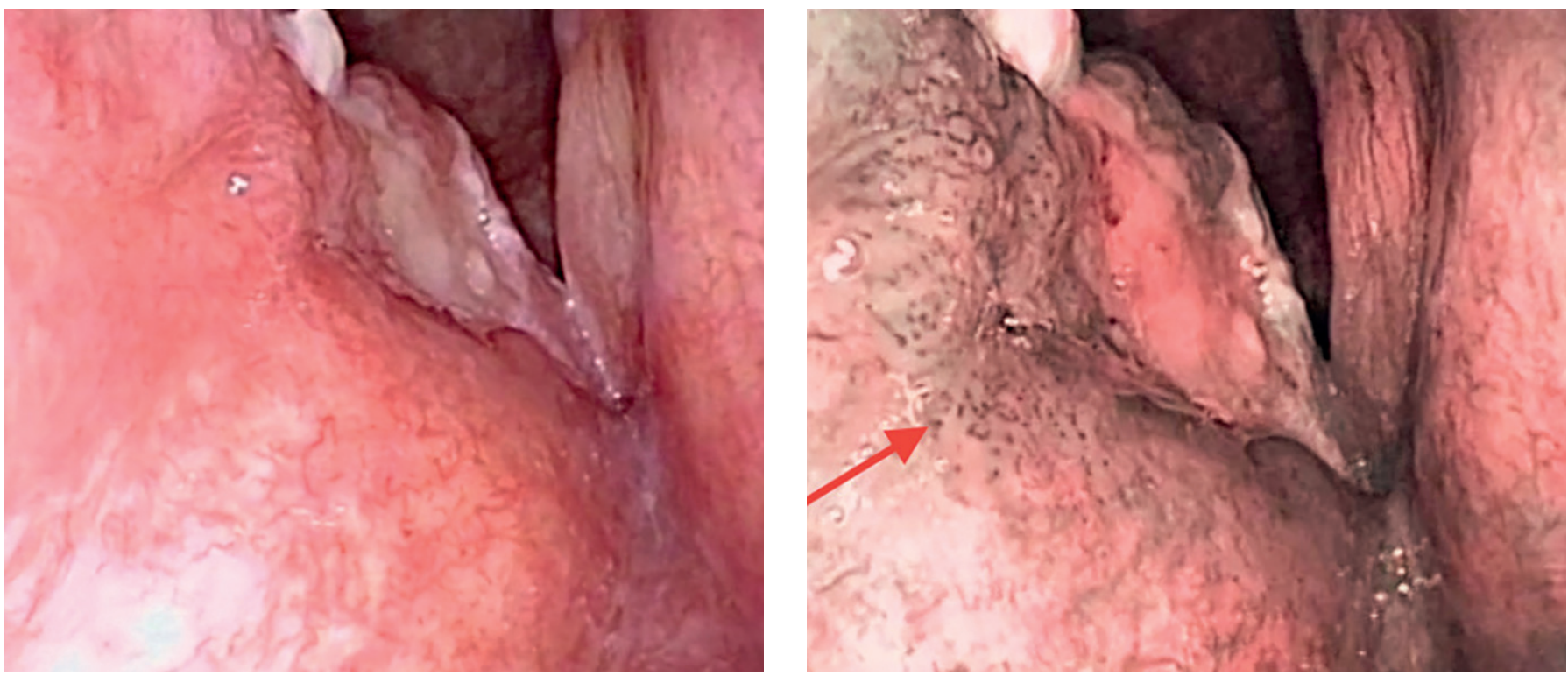

Fig. 2 Squamous cell carcinoma of right vocal cord and vestibular fold: (A) in conventional WLE, (B) in NBI malignant type of vascularization (the red arrow is pointing to the affected area with IPCL type Va).

\section{MATERIAL AND METHODS}

The presented study was conceived as a prospective study. Patients with clinically suspicious laryngeal or hypopharyngeal lesion that presented to the Department of Otorhinolaryngology and Head and Neck Surgery in Hradec Králové (Czech Republic) from October 2013 to December 2019 were recruited. A total of 589 patients were investigated using conventional WLE and NBI endoscopy with high-definition TV (HDTV NBI) from 10/2013 to $12 / 2019$. Patients were divided into two groups based on preoperative NBI examination (group A $=345$ patients) and intraoperative NBI examination (group B $=244$ patients). All suspected lesions were graded to 5 types of $\mathrm{Ni}$ classification and correlated with histopathologic analysis.

The main reasons for referral were patients with chronic laryngitis, hoarseness or voice changes of unknown etiology lasting more than 3 weeks, benign - appearing lesions (polyps, cysts, vocal cord granulomas), leukoplakias, erythroplakias, macroscopic tumorous lesions of vocal cords or laryngeal papillomatosis. During the consultation in out-patient department (group A), flexible laryngoscopy was performed. After application of mesocain gel (trimecainhydrochloridum carbethopendeciniibromidum $10 \mathrm{mg} / \mathrm{g}$ ), flexible videolaryngoscope was inserted through the nasal cavity. For local anesthesia of larynx we used lidocaine spray (10\% lidocainum) with specially curved applicator that was applied transorally. Flexible endoscopy was performed with an Olympus ENF-VH or ENF-VQ $3.9 \mathrm{~mm}$ flexible endoscope connected to an Elvis Exera III CV 170 light source (Olympus Medical System, Tokyo, Japan) while patients were awake and seated. Each patient was examined by conventional WLE followed by NBI mode where the lesions were assessed in real time and captured. In conventional WLE we evaluated macroscopic characteristics of the lesion (chronic laryngitis, cyst, polyp, granuloma, Reinke oedema, leukoplakia, erythroplakia, papilloma, exophytic lesion) and the extent and spreading of the lesion (Figure 2). In NBI mode we described changes in microvascularization graded according to $\mathrm{Ni}$ classification. Any lesions considered being precancerous were graded IV, lesions suspected from malignancy were given grade $\mathrm{V}(\mathrm{Va}, \mathrm{Vb}$ or $\mathrm{Vc}$ ). Endoscopic examination was performed only by three physicians. A clinically indicated biopsy was taken under general anaesthesia in the operating room. The biopsy specimens were fixed in $10 \%$ formalin and sent for histopathological examination. The tissue was routinely processed, stained with hematoxylin and eosin and examined by an experienced head and neck pathologist. Histopathological diagnoses were then compared to their respective Ni classification (Table 1). Based on this, sensitivity and specificity of NBI using the Ni classification for detection of laryngeal and hypopharyngeal cancer were calculated. Exclusion criteria were allergy to local anaesthetics, previous laryngeal or hypopharyngeal cancer, or inability to undergo flexible endoscopy due to increased gag reflex.

In group B patients underwent examination in general anaesthesia in the operating theatre (direct laryngoscopy or hypopharyngoscopy). After the endotracheal intubation, all anatomical sites were endoscopically evaluated by WLE and NBI using rigid $0^{\circ}$ and $30^{\circ}$ angled telescopes (Olympus Visera Pro system CV-170 with Olympus OTV S 7 camera head). Attention was paid to the superficial extension of the primary lesion with special emphasis to the additional suspected areas in the entire larynx and hypopharynx. The principles of classification of NBI findings and correlation with histopathological results were identical to the group A.

The SPSS version 8.0.4 statistical software package was used for statistical analysis. Sensitivity, specificity, positive predictive value, and negative predictive value for detection of laryngeal and hypopharyngeal cancer were calculated. 


\section{RESULTS}

From October 2013 to December 2019, a total of 345 patients were included in group A. There were 248 (71.9\%) males and 97 (28.1\%) females (male to female ratio $2.5: 1$ ) aged 34 to 79 years (median 53; mean $58.4 \pm 16.5$ ).

In group B total of 244 patients were included. There were $169(69.3 \%)$ males and 75 (30.7\%) females (male to female ratio $2.3: 1$ ) aged 27 to 81 years (median 59; mean $56.2 \pm 14.5)$. Detailed personal data are summarized in Table 2.

Tab. 2 Age, sex and comorbidities in two groups of patients.

\begin{tabular}{|l|l|l|}
\hline Characteristics & Group No. I & Group No. II \\
\hline men & $72.4 \%$ & $69.3 \%$ \\
\hline women & $27.6 \%$ & $30.7 \%$ \\
\hline smokers & $65.5 \%$ & $71.7 \%$ \\
\hline non-smokers & $24.1 \%$ & $19.7 \%$ \\
\hline stop-smokers & $10.4 \%$ & $8.6 \%$ \\
\hline age & $64.3 y(43-82)$ & $56.3 y(27-81)$ \\
\hline hoarseness & $79.3 \%$ & $79.1 \%$ \\
\hline chronic laryngitis & $23.3 \%$ & $29.1 \%$ \\
\hline 1 comorbidity $<60 \mathrm{y}$ & $74.1 \%$ & $65.8 \%$ \\
\hline$\geq 2$ comorbidities $>60 \mathrm{y}$ & $77.6 \%$ & $72.2 \%$ \\
\hline Extraesophageal reflux & $17.2 \%$ & $23.4 \%$ \\
\hline
\end{tabular}

In group $\mathrm{A}$, we detected pathological findings in $324 / 345$ (94\%) patients when using the conventional WLE, especially chronic laryngitis (16\%), leukoplakia (22\%), erythroplakia (4\%), exophytic tumour (35\%), papilloma (15\%) and Reinke oedema (8\%). Using NBI endoscopy, larger extent of the lesion was visualized in $36 \%$ and new lesions not detected in white light in $6 \%$ cases $(\mathrm{Ta}-$ ble 3). Histopathological examination was made in $85 \%$

\section{A}

Benign type of vascularisation

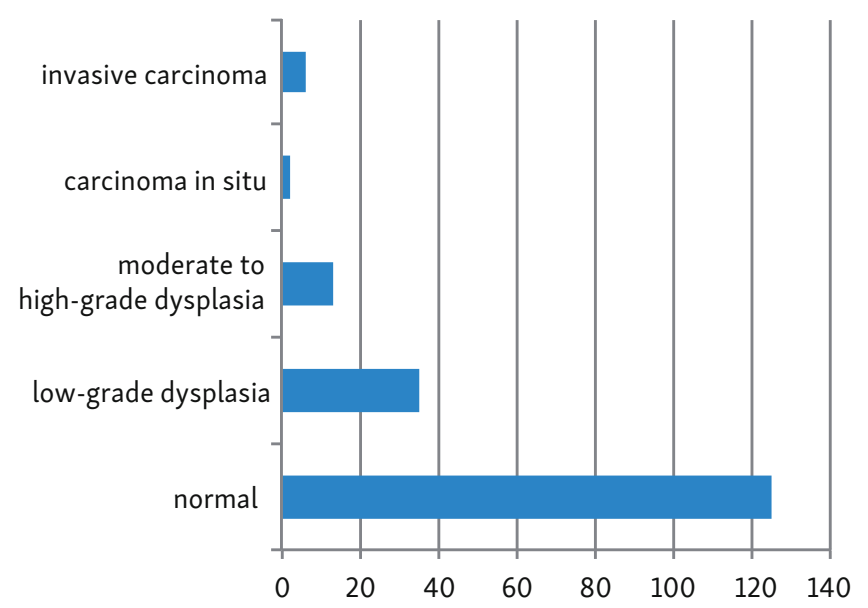

Tab. 3 Overview of own results (Statistics: software SPSS version 8.0.4).

\begin{tabular}{|l|l|l|}
\hline NBI examination & Group No. I & Group No. II \\
\hline Larger extent of lesion in NBI & $36.0 \%$ & $22.1 \%$ \\
\hline New lesions in NBI & $6.0 \%$ & $17.6 \%$ \\
\hline Sensitivity & $86.2 \%$ & $90.8 \%$ \\
\hline Specificity & $90.9 \%$ & $94.7 \%$ \\
\hline Positive predictive value & $75.8 \%$ & $93.6 \%$ \\
\hline Negative predictive value & $95.2 \%$ & $86.5 \%$ \\
\hline $\begin{array}{l}\text { Overall agreement } \\
95 \% \text { IS }\end{array}$ & $\begin{array}{l}86.84 \% \\
(76.4-93.4 \%)\end{array}$ & $\begin{array}{l}89.64 \% \\
(79.4-94.6 \%)\end{array}$ \\
\hline $\begin{array}{l}\text { Қ index (p<0.001) } \\
95 \% \text { IS }\end{array}$ & $\begin{array}{l}0,76 \\
(0.54-0.96)\end{array}$ & $\begin{array}{l}0.8461 \\
(0.8274-0.9654)\end{array}$ \\
\hline
\end{tabular}

(293 patients). If we assumed benign mucosal vascularization in NBI, normal finding was reported by pathologist in $69.1 \%$ lesions, dysplastic changes in $26.4 \%$, carcinoma in situ in $1.2 \%$ and invasive spinocellular carcinoma in $3.3 \%$ (Graph 1A). If we assumed malignant mucosal vascularization in NBI, an invasive spinocellular carcinoma was proven in $46.4 \%$ cases, carcinoma in situ in $8.9 \%$, moderate to high-grade dysplasia in $30.4 \%$, low-grade dysplasia in $5.4 \%$, and chronic inflammatory changes without dysplasia in $8.9 \%$ cases (Graph 1B).

Character of mucosal vascularization (IPCL) in NBI was correlated with histopathological examination. Sensitivity of NBI in distinction of benign hyper- and parakeratosis, low-grade dysplasia, moderate to high-grade dysplasia from advanced precancerous stages (carcinoma in situ) and invasive spinocellular carcinoma (SCC) was $86.2 \%$ (95\% IS: $65.4-95.2$ ), while specificity was $90.9 \%$ (95\% IS: 70.6-94.1). Overall agreement was $86.84 \%$ (95\% IS: 76.4-93.4). According to Kappa index $\mathrm{K}=0.76$ (95\% IS: $0.54-0.96)(p<0.001)$, we proved statistically significant agreement between NBI and histopathological examination (Table 3).

\section{B Malignant type of vascularisation}

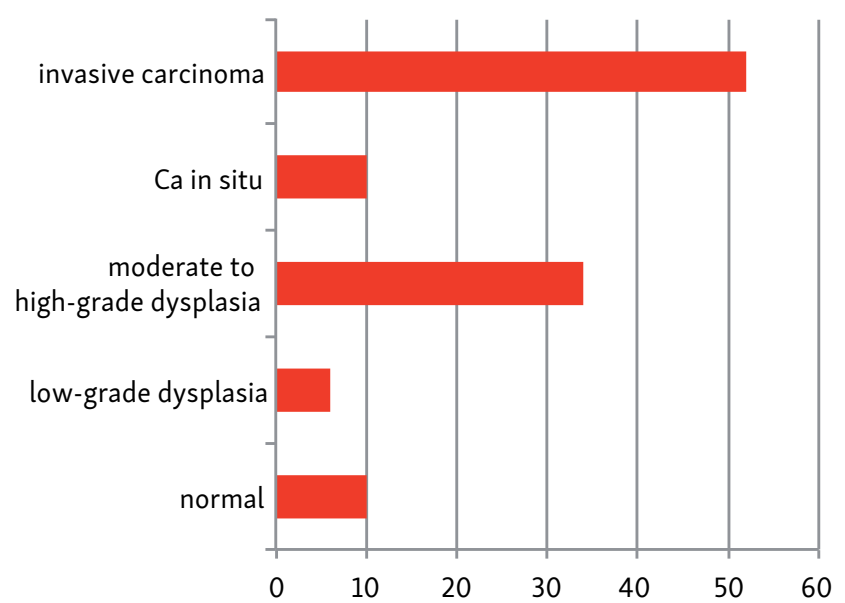

Graph 1 Comparison of vascular pattern of intraepithelial papillary capillary loops in NBI (according to Ni et al. 2011) and the results of histopathological analysis in group A. (A) benign type of vascularization in NBI, (B) malignant type of vascularization in NBI. 


\section{A Benign type of vascularisation}

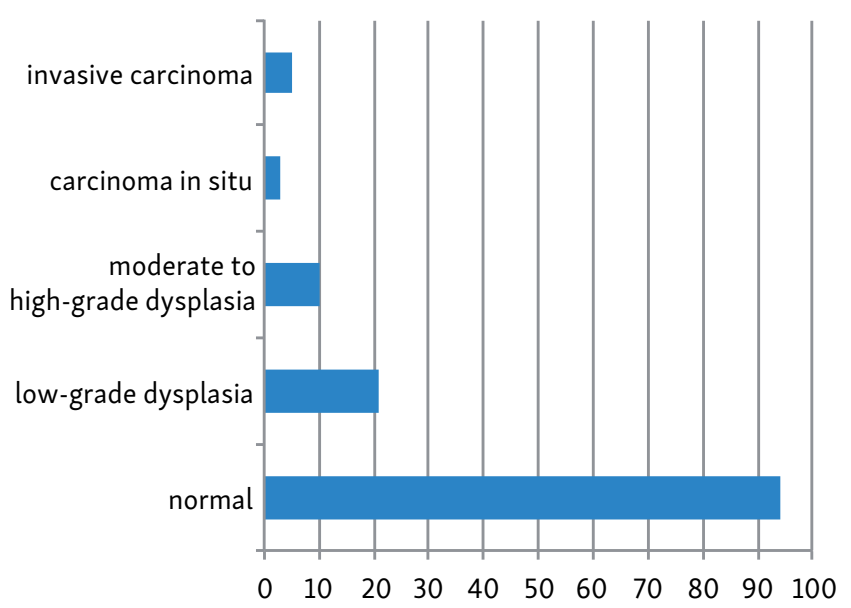

B Malignant type of vascularisation

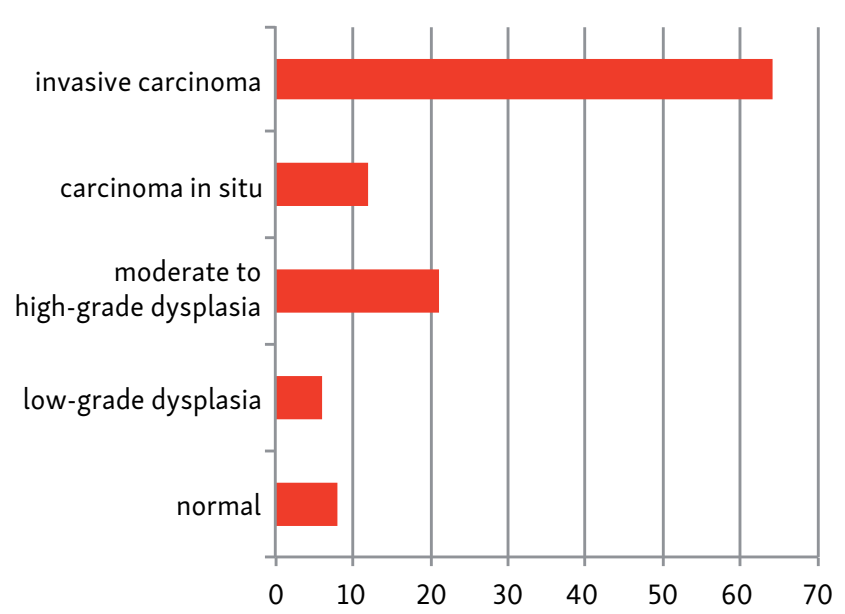

Graph 2 Comparison of vascular pattern of intraepithelial papillary capillary loops in NBI (according to Ni et al. 2011) and the results of histopathological analysis in group B. (A) benign type of vascularization in NBI, (B) malignant type of vascularization in NBI.

In group B, larger extent of the lesion was visualized in $22.1 \%$ and new lesions not detected in conventional white light in $17.6 \%$ cases (Table 3). Histopathological examination verified benign findings (hyperkeratosis, parakeratosis, chronic inflammatory changes, polyps, cysts and granulomas) in $37.3 \%$ of cases. Recurrent respiratory papillomatosis was present in $14.3 \%$ and low-grade dysplastic changes in $8.6 \%$ of cases (Graph $2 \mathrm{~A}$ ). As regards precancerous and malignant changes, we proved high-grade dysplasia in $13.9 \%$, carcinoma in situ in $3.3 \%$ and invasive SCC in $22.5 \%$ of patients (Graph $2 B$ ). Statistical analysis confirmed the sensitivity of $90.8 \%$ (95\% IS: $82-96)$ and specificity of $94.7 \%$ (95\% IS: 79.4-94.6). Overall agreement was $89.64 \%$ (95\% IS: 79.4-94.6). According to Kappa index $\mathrm{K}=0.8461$ (95\% IS: $0.8274-0.9654)(\mathrm{p}<0.001)$ we found statistically significant agreement between NBI and histopathological examination (Table 3).

\section{DISCUSSION}

Patients with UADT tumors are routinelly examined by conventional white light endoscopy. It is the most commonly used method to evaluate superficial spreading of SCC. In early stages of cancerogenesis, the tumors of UADT are hardly distinguished from normal tissue and usually not identified by conventional WLE (5). The growth of epithelial malignant tumors leads to escalated neoangiogenesis, which may be observed in endoscopic practice as changes in the IPCL arrangement, diameter, shape and as a loss of regularity (1). Technological improvement in endoscopic methods is the key for early detection of these changes. Alteration of microvascular architecture becomes more visible in high-grade dysplastic changes in comparison to low-grade dysplastic changes (1).

NBI is easily applicable and well tolerated in local anesthetics in out-patient department. Great practical significance is identification of new suspicious lesions. We found out larger extent of suspicious lesions in $36 \%$ of patients, and in $6 \%$ of cases we identified new lesions that were not be visible by conventional white light endoscopy. Piazza et al. (2010) proved larger extent of lesions in $26 \%$ and new lesions not visible in WLE in 9\% (8).

Piazza et al. (2012) also proved the benefit of pre-operative using of NBI with detection up to 20 lesions not visible by white light endoscopy. The diagnostic benefit of NBI in better definition of neoplastic superficial spreading is substantially increased with using HDTV NBI (from $20.8 \%$ to $42.7 \%$ ) (9).

Clinical application of NBI endoscopy requires a special practice and defined "learning curve". Correct recognition and diagnostics of lesions is long-lasting process. Villaseca et al. (2017) demonstrated, that accuracy of detection of malignant lesions increased with longer-lasting practical experience of the examiner and he recommends at least 200 examinations for correct identification of vascular changes (10). Piazza et al. (2010) demonstrated that 13.7\% from 58 positive findings in NBI were false positive, if $70 \%$ of the findings have been diagnosed during the first three months of using NBI. The advantage of NBI examination in out-patient department is possibility of data recording and fast video analysis in a short time (8).

Piazza et al. (2010) assessed 279 patients with laryngeal cancer and reported a sensitivity of only $61 \%$ using flexible NBI alone, but the sensitivity increased substantially to $98 \%$ and specificity to $90 \%$ when NBI was coupled with HDTV (8). Shoeffel-Havakuk et al. (2017) compared NBI with WLE for the diagnosis of laryngeal cancer and reported a sensitivity of $58.6 \%$ when using NBI. Sensitivities reported in this study were based on three expert assessments of NBI and WLE images, not histopathological diagnosis like in other studies (11). Chang et al. (2016) consider $\mathrm{NBI}$ as an effective technique with high diagnostic accuracy (98.9\%), sensitivity (97.2\%) and specificity (100\%), and therefore recommend using flexible endoscopy with NBI and taking biopsy in out-patient department. Diagnostic accuracy was not affected by size, localization, "learning" or history of head and neck cancer (12). In recent 
publication by Hosono et al. (2019), sensitivity using HDTV NBI in laryngeal lesions was $84.4 \%$ (13). Others recent studies have demonstrated higher sensitivities of $>90 \%$ when using HDTV NBI in laryngeal and hypopharyngeal cancer (14).

Young and colleagues (2015) reported a sensitivity of $91.2 \%$ in 23 malignant lesions (15). Sakthivel et al. (2018) confirmed sensitivity up to $100 \%$ if WLE and NBI were combined (16). In study by Rzepakowska et al. (2018) the sensitivity was up to $98.8 \%$ with the HDTV NBI in diagnostics of precancerous and malignant laryngeal lesions (17). In our study we achieved sensitivity $86.2 \%$ and specificity 90.6\% and according to Kappa index there was substantial agreement between NBI and histopathological results $(\mathrm{K}=0.76 ; \mathrm{p}<0.001)$.

NBI has also limitations in preoperative diagnosis. To obtain a valid interpretation it is necessary to keep minimal distance between distal part of endoscope to the examined mucosa ( $3 \mathrm{~mm}$ and less) which can be difficult in patients with strong gag reflex even after using local anaesthetics. Another disadvantage is the impossibility to assess vertical extent of lesion. The cause of false negative findings might be due to hyperkeratosis making the assessment of pathological vascularization impossible ( 3 , 18). In our study there were two patients with verrucous SCC where it was not possible to assess IPCL. In one patient with adenoid cystic carcinoma superficial pathological vascularization was not identified due to submucosal spreading of the tumour. Assessment of leukoplakia in NBI is controversial as well, described by a well-known phenomenon - the "umbrella effect", which reflects the submucosal vascular pattern being hidden under the hyperkeratotic plaque (Klimza et al. 2017) (19). To overcome the "umbrella effect", the NBI was used to categorise submucosal vascular pattern surrounding the plaque, as illustrated in Figure 3B (Klimza et al. 2017, Stanikova et al. 2017) $(18,19)$. Laryngeal recurrent respiratory papillomatosis (RRP) is another problematic lesion that can be confused with SCC (Figure 4). In our study we found

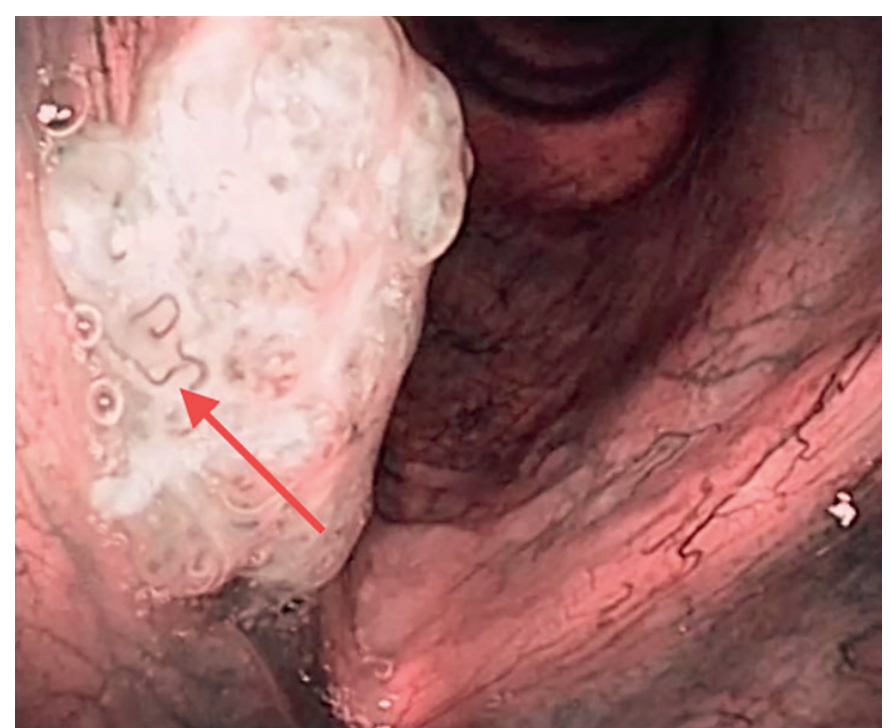

Fig. 3 Squamous cell carcinoma of right vocal cord (the red arrow is showing pathological vascularization - irregular, tortuous, line-like shapes - IPCL type Vb).

27 patients with RRP (8.3\%), 3 cases were initially suspected from malignancy and proved to be false positive. However, if RRP is suspected other synchronous papillomatous lesions (Jackowska et al. 2018) can be detected in NBI. In our study NBI revealed other lesions not visible in WLE in $14 / 27(51.8 \%)(20)$.

In peroperative using of HDTV NBI we demonstrated significant difference in comparison with conventional WLE. We identified larger extent of lesions in $22.1 \%$ and new suspicious lesions not visible by WLE in $17.6 \%$. The sensitivity was $90.8 \%$, specificity $94.7 \%$ and according to Kappa index $(\mathrm{K}=0.8461 ; \mathrm{p}<0.001)$ almost perfect agreement between NBI and histopathological examination has been proven. We had low amount of false positive results and high number of true negative results, and therefore the specificity is so remarkable.
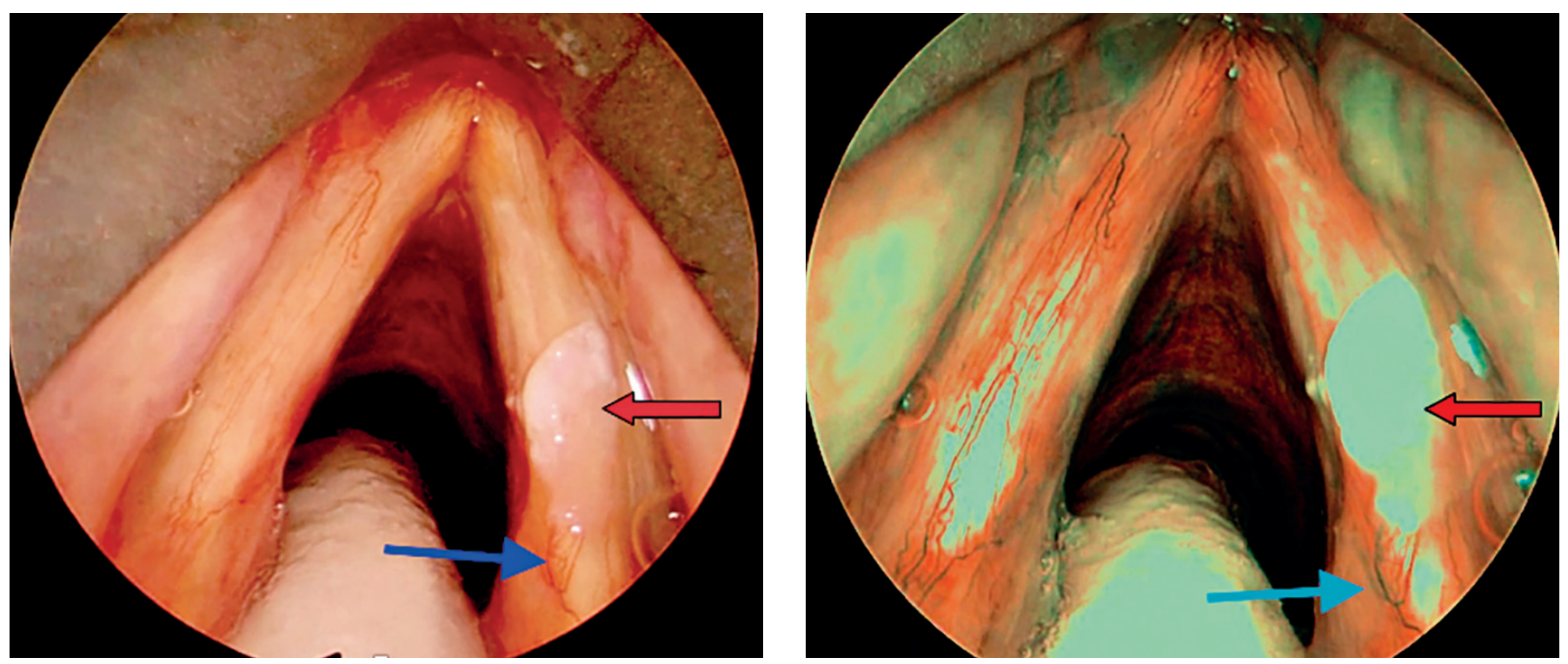

Fig. 4 Leukoplakia on the right vocal cord - intraoperative finding in direct laryngoscopy: (A) in conventional WLE, (B) in NBI (the red arrow is showing the lesion, the blue arrow is pointing to the surrounding IPCL type II). 
It is difficult to determine margins between physiological and pathological findings when taking "ideal" biopsy in conventional WLE. There are many studies that evaluate positive intraoperative effect of NBI to reduce the number of positive resection margins $(21,22)$. They proved benefit of NBI in intraoperative diagnostics for better visualization of margins in superficial spreading lesions what is important in endoscopic surgery. Garofollo et al. (2015) proved significant reduction of positive resection margine using NBI (from $23.7 \%$ to $3.6 \%$ ) in patients with early glottis carcinoma (cordectomy type I and II) (21). Piazza et al. (2010) confirmed the significance of NBI in 26 laryngeal tumors where HDTV NBI used intraoperatively led to enlargement of resection margins (23). In a recent study performed by Klimza et al. (2019) pathological lesions not visible in WLE were identified in $13.6 \%$ and histopathologically confirmed in $88.8 \%$ (33.3\% invasive SCC, $66.6 \%$ high-grade dysplasia and carcinoma in situ) (22). Orita et al. (2008) confirmed intraoperative benefit of NBI during the endoscopic hypopharyngeal surgery (24).

\section{CONCLUSION}

The results in all above mentioned published studies as well as our study confirmed that NBI is a reliable method in early detection of precancerous and malignant changes, for discriminating between benign and malignant patterns in prehistological diagnosis.

Great practical significance is identification of new suspicious lesions not visible by conventional white light endoscopy that we proved preoperatively in $6 \%$ and intraoperatively in $17.6 \%$ of cases. We confirmed low amount of false positive results and high number of true negative results, and therefore the specificity was so remarkable. Especially in peroperative using of HDTV NBI we demonstrated almost perfect agreement between NBI and histopathological examination.

NBI has been proved to be a "gold standard" and routine diagnostic tool for assessment of laryngeal and hypopharyngeal pathology.

\section{CONFLICT OF INTEREST}

The authors declare that there is no actual or potential conflict of interest in relation to this article.

\section{FINANCIAL DISCLOSURE}

The authors declare that this study has received no financial support.

\section{REFERENCES}

1. Ahmazada S, Tseros E, Sritharan N, et al. The value of narrowband imaging using the Ni classification in the diagnosis of laryngeal cancer. Laryngoscope Investig Otolaryngol 2020; 5(4): 665-71.

2. Lukes P, Zabrodsky M, Lukesova E, et al. The role of NBI HDTV Magnifying endoscopy in the Prehistologic Diagnosis of Laryngeal Papil- lomatosis and Spinocellular Cancer. BioMed Res Int 2014; article ID 285486.

3. Lukes P, Zabrodsky M, Plzak J, et al. Narrow Band Imaging (NBI) Endoscopic Method for Detection of Head and Neck Cancer. Endoscopy. S. Amornyotin 2013.

4. Ni XG, Wang GQ. The Role of Narrow Band Imaging in Head and Neck Cancers. Curr Oncol Rep 2016; 18(2): 1-7.

5. Abdullah B, Abdull Rasid NS, Mat Lazim N, et al. Ni endoscopic classification for Storz Professional Image Enhancement System (SPIES) endoscopy in the detection of upper aerodigestive tract (UADT) tumours. Sci Rep 2020; 10(1): 1-7.

6. Ni XG, He S, Xu ZG, et al. Endoscopic diagnosis of laryngeal cancer and precancerous lesions by narrow band imaging. J Laryngol Otol 2011; 125(3): 288-96.

7. Arens C, Piazza C, Andrea M, et al. Proposal for a descriptive guideline of vascular changes in lesions of the vocal folds by the committee on endoscopic laryngeal imaging of the European Laryngological Society. Eur Arch Otorhinolaryngol 2016; 273(5): 1207-14.

8. Piazza C, Cocco D, De Benedetto L, et al. Narrow Band Imaging and High Definition Television in the assessment of laryngeal cancer: a prospective study on 279 patients. Eur Arch Otorhinolaryngol 2010; 267(3): 409-14.

9. Piazza C, Del Bon F, Peretti G, et al. Narrow band imaging in endoscopic evaluation of the larynx. Curr Opin Otolaryngol Head Neck Surg 2012; 20(6): 472-6.

10. Villaseca I, Valls - Mateus M, Nogués A, et al. Usefullness of office examination with narrow band imaging for the diagnosis of head and neck squamous cell carcinoma and follow - up of premalignant lesions. Head Neck 2017; 39(9): 1854-63.

11. Shoffel-Havakuk H, Lahav Y, Meidan B, et al. Does narrow band imaging improve preoperative detection of glottic malignancy? A matched comparison study. Laryngoscope 2017; 127(4): 894-99.

12. Chang C, Lin WN, Hsin LJ, et al. Reliability of office-based narrow-band imaging-guided flexible laryngoscopic tissue samplings. Laryngoscope 2016; 126 (12): 2764-69.

13. Hosono H, Katada C, Okamoto T, et al. Usefulness of narrow band imaging with magnifying endoscopy for the differential diagnosis of cancerous and noncancerous laryngeal lesions. Head Neck 2019; 41(8): 2555-60.

14. Klimza H, Jackowska H, Piazza C, et al. The role of intraoperative narrow-band imaging in transoral laser microsurgery for early and moderately advanced glottic cancer. Braz J Otorhinolaryngol 2019; 85(2): 228-36.

15. Young CK, Lin WN, Lee LY. Laryngoscopic characteristics in vocal leukoplakia: inter-rater reliability and correlation with histology grading. Laryngoscope 2015; 125(2): 62-66.

16. Sakthivel P, Sikka K, Thakar A, et al. Role of narrow band imaging in the diagnosis of laryngeal lesions: pilot study from India. Indian J Cancer 2018; 55(3): 242-7.

17. Rzepakowska A, Sielska-Badurek E, Cruz R, et al. Narrow band imaging versus laryngovideostroboscopy in precancerous and malignant vocal fold lesions. Head Neck 2018; 40(5): 927-36.

18. Stanikova L, Satankova J, Kucová H, et al. The role of narrow-band imaging (NBI) endoscopy in optical biopsy of vocal cord leukoplakia. Eur Arch Otorhinolaryngol 2017; $274(1): 355-9$.

19. Klimza H, Jackowska J, Tokarski M, et al. Narrow-band imaging (NBI) for improving the assessment of vocal fold leukoplakia and overcoming the umbrella effect. Plos One 2017; 12(6): 1-7.

20. Jackowska J, Klimza H, Winiarski P, et al. The usefulness of narrow band imaging in the assessment of laryngeal papillomatosis. Plos One 2018; 13(10); 1-9.

21. Garofolo S, Piazza C, Bon FD, et al. Intraoperative Narrow Band Imaging Better Delineates Superficial Resection Margins During Transoral Laser Microsurgery for Early Glottic Cancer. The Annals of Otology, Rhinology and Laryngology 2015; 124(4): 294-8.

22. Klimza H, Jackowska H, Piazza C, et al. The role of intraoperative narrow-band imaging in transoral laser microsurgery for early and moderately advanced glottic cancer. Braz J Otorhinolaryngol 2019; 85(2): 228-36.

23. Piazza C, Cocco D, De Benedetto L, et al. Narrow Band Imaging and High Definition Television in the assessment of laryngeal cancer: a prospective study on 279 patients. Eur Arch Otorhinolaryngol 2010; 267(3): 409-14.

24. Orita Y, Kawabata K, Mitani H, et al. Can Narrow-band Imaging be used to determine the surgical margins of superficial hypopharyngeal cancer? Acta Med Okayama 2008; 62 (3): 205-8. 